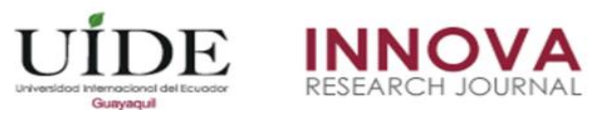

INNOVA Research Journal, ISSN 2477-9024

(Junio, 2018). Vol. 3, No.6 pp. 168-176

DOI: https://doi.org/10.33890/innova.v3.n6.2018.897

URL: http://revistas.uide.edu.ec/index.php/innova/index

Correo: innova@uide.edu.ec

\title{
La importancia del uso del material didáctico para la construcción de aprendizajes significativos en la educación inicial
}

\section{The importance of the use of didactic material for the construction of significant learning in initial education}

\author{
Zila Isabel Esteves Fajardo \\ Universidad de Guayaquil, Ecuador \\ Norma Garcés Garcés \\ Universidad de Guayaquil, Ecuador \\ Verónica Narcisa Toala Santana \\ Elizabeth Eunice Poveda Gurumendi \\ Colegio Emblemático Guayaquil, Ecuador \\ Autor para correspondencia: zilaisabelesteves@ hotmail.es, Norma.garcesg@ug.edu.ec, \\ veritont@hotmail.com, lizlpz30@yahoo.com \\ Fecha de recepción: 25 de Enero de 2018 - Fecha de aceptación: 26 de Junio de 2018
}

Resumen: El presente trabajo de investigación analizó la situación de las escuelas de Ecuador respecto al uso del material didáctico y a la ejecución de las varias estrategias educativas. Quisiera darle valor a la palabra "innovación" y que reciba una acepción positiva en el ámbito escolar; ya que, más que suplantar, quisiera incursionar, sugestionar y explorar todos los materiales didácticos que ayuden en el aprendizaje significativo. No se trata de eliminar y cambiar el "tradicional" material didáctico de uso en las escuelas, por otros tal vez mejores y más dinámicos; sino que le sumen a éste y que sean vistos como "pasos positivos", nacidos como una fusión entre lo nuevo y lo viejo, hacia y para una nueva sociedad. De hecho, no debemos apartarnos de la realidad cada vez más cambiante, sino involucrarnos a ella y tomar lo positivo de manera consciente y sabia. Se tiende, por lo general, a ser reacios a lo que se concibe como nuevo, pero es bueno, creativo y útil acoger nuevas técnicas o nuevos conocimientos. Se debe aprender a ver los nuevos conocimientos como la parte enriquecedora de nuestra cultura. De todo lo nuevo se puede aprender y de todo lo viejo se puede mejorar y crear algo mejor. Es, por tanto, indispensable que se abran nuevos horizontes para acoger las pautas que nos brinda el sistema escolar sin miedo a errores o equivocaciones. El trabajo realizado, a través de la investigación de campo, llegó a la conclusión que más recursos didácticos significa tener más posibilidades de desarrollo.

Palabras Claves: material didáctico; innovación; sistema escolar; fusión; desarrollo

Abstract: This research work analyzed the situation of the Ecuador's schools regarding the use of didactic material and the implementation of various educational strategies. I would like to give value to the word "innovation" and to receive a positive meaning in the school environment. Therefore, I would like that the word "innovation" to be given a positive meaning in the school environment. It therefore wants more than supplanting, incursing and managing the use of other didactic materials that help in meaningful learning. It is not a question of eliminating and changing the "traditional" didactic material of use in schools, for others perhaps better and more dynamic; 
But that they add to this and that they are seen as "positive changes", born as a fusion between the old and new, towards and for a new society. In fact, we must not deviate from the ever-changing reality, but involve ourselves and take the positive in a conscious and wise way. It tends, generally, to be reluctant to what is conceived as new, but it is good, creative and useful to welcome new techniques or new knowledge. You must learn to see the changes as the enriching part of our culture. Everything new can be learned and everything old can be improved and create something better. It is, therefore, indispensable that new horizons are opened to welcome the guidelines provided by the school system without fear of errors or mistakes. The work done through the field research, came to the conclusion that more teaching resources means have more possibilities of development.

Key Words: didactic material; innovation; school system; fusion; development

\section{Introducción}

El primer punto clave es que, hoy en día, la palabra sola no basta, y creo que nunca ha bastado, para transmitir un aprendizaje; ya que se necesita experimentar y explorar; sobre todo en esta sociedad cambiante. La llamo cambiante porque los niños de ahora, non son los mismos de hace unos cuantos años atrás. Hay que estar al paso del tiempo y seguir su ritmo. Nacen niños, se podría decir: más activos, más curiosos, más exploradores. Efectivamente, todo evoluciona; de consecuencia, se necesita estar al día con nuevas innovaciones o mejoramientos de materiales didácticos ya en uso. De hecho, no solo ha cambiado la sociedad, sino también el modo de enseñar. Antes se hablaba y se practicaba el modelo pedagógico tradicional, conductista; ahora, está tomando la revancha el modelo constructivista que ve al niño como el actor de su propio aprendizaje a partir de sus conocimientos previos y les da un realce fundamental a sus experiencias.

Según (Guerrero \& Idrovo, 2010), en su publicación nos indican: El estudio sobre material didáctico para niños/as en etapa inicial no es algo nuevo, se llevan años de investigaciones y análisis para ir perfeccionándolo y adaptándole de manera adecuada a los cambios culturales y sociales de acuerdo con el tiempo en que se vive.

Para María Montessori: «El niño, con su enorme potencial físico e intelectual, es un milagro frente a nosotros. Este hecho debe ser transmitido a todos los padres, educadores y personas interesadas en niños, porque la educación desde el comienzo de la vida podría cambiar verdaderamente el presente y futuro de la sociedad. Tenemos que tener claro, eso sí, que el desarrollo del potencial humano no está determinado por nosotros. Solo podemos servir al desarrollo del niño, pues este se realiza en un espacio en el que hay leyes que rigen el funcionamiento de cada ser humano y cada desarrollo tiene que estar en armonía con todo el mundo que nos rodea y con todo el universo».

En ese entonces, ya lo decía ella que el niño necesitaba guías que lo encaminen para expresar lo mejor que había en él. Pasó largo tiempo para que finalmente se propusieran a crear una nueva metodología de enseñanza.

En efecto, hoy en día, nacen como espacios didácticos los rincones del aula. La organización de las clases por "rincones" es una propuesta metodológica que hace posible la participación de los niños /as en la construcción de sus conocimientos. Sin embargo, datos 
afirman que no todas las instituciones ecuatorianas cumplen con el currículo y, por ende, no se les brinda a los niños/as el abanico de grandes oportunidades de aprendizajes que se han implementado.

Desde el 2014, el Ministerio de Educación puso en vigencia el Currículo de Educación Inicial para orientar los procesos educativos, formales y no formales, dirigidos a las niñas y niños menores de cinco años.

Según (Alvarado, 2017), nos indica: “El currículo de Educación Inicial define a las experiencias de aprendizaje como un conjunto de vivencias y actividades desafiantes, intencionalmente diseñadas por el docente, que surgen del interés de los niños produciéndoles gozo y asombro, teniendo como propósito promover el desarrollo de las destrezas que se plantean en los ámbitos de aprendizaje y desarrollo."

El Currículo también establece determinadas características que deben tener las experiencias de aprendizaje, entre las cuales cabe citar a las siguientes:

- Garantizar la participación de todos los niños.

- Respetar el ritmo de aprendizaje, ya que cada niño aprende en tiempos distintos y con diferentes estilos.

- Tener pertinencia cultural y contextual.

- Facilitar la interacción positiva entre los niños y también con los adultos; es decir, establecer una relación libre de tensiones, donde los niños se encuentren inmersos en sus actividades gracias al disfrute que estas provocan.

- Propiciar la indagación y reflexión como procesos significativos.

A partir de lo que establece el Currículo de Educación Inicial, se entiende que las experiencias de aprendizaje NO SON:

- Actividades didácticas escolarizadas.

- Actividades rígidas con una sola respuesta, que todos los niños deben reproducir.

- Actividades mecánicas que se "hacen por hacer", sin un motivo o finalidad real.

- Hojas de trabajo prediseñadas para que los niños ejecuten consignas mecánicas y repetitivas. (Ministerio de Educación, Currículo Educación Inicial, pp.23).

Justamente, a estas últimas 4 actividades, se siguen rigiendo muchas escuelas. Se trata de un trabajo muy monótono que lleva al niño al aburrimiento total y lo aparta del crear por si solo su aprendizaje significativo por medio de la experiencia. Puede ser que, por facilitarse el trabajo, lo lleven a cabo de esta manera y mientras que la institución se beneficia, los niños/as son los perjudicados; esto, para las instituciones privadas.

Por otro lado, si bien el antiguo gobierno realizaba mucha propaganda por la educación, sosteniendo que había mejorado, que se invertía y se apostaba más por ella, incluso se llegó a decir que los la UNESCO consideraba Ecuador como el país de Latino América más desarrollado en Educación, Reduca (Red Latinoamericana por la Educación) sostiene todo lo contrario. Según esta organización, en Ecuador se invierte solo un 0,2\% del PIB en Educación Preescolar, la mitad que el promedio de Latino América. 
El dato fue dado en el 2016, es muy reciente y, de hecho, la realidad no es como exactamente la manifiestan y en la investigación lo pude corroborar. Si el Gobierno invirtiera un poco más en la Educación Inicial, otra fuera la importancia que se le diera a esta etapa muy importante y significativa de la vida de todos los niños/as.

\section{Hipótesis}

El uso del material didáctico influye significativamente en el aprendizaje de los niños/as.

\section{Objetivo general}

Analizar de qué manera incide el no usar en lo absoluto o en parte el material a disposición y los que carecen completamente de ellos.

\section{Importancia del problema}

El material didáctico es de suma importancia para el desarrollo de los niños/as en esta edad, pues la mejor manera de aprender es mediante el juego y la diversión a través del uso de material concreto, así se logra que los pequeños se involucren de manera interactiva a la hora de aprender; considerándose una etapa fundamental y determinante para el resto de los años venideros. Estas experiencias del niño/a con distintos estímulos permiten que avance su desarrollo, por ello el uso de material didáctico se hace cada vez más necesario para la enseñanza de los niños/as, favorece su observación y sus habilidades para la toma de decisiones. Los niños/as alcanzan un nivel de creatividad sorprendente dado que motiva mentes más sanas, democráticas, cambia la forma de ver y asumir la vida, formándose así la disciplina y responsabilidad hacia el autoaprendizaje.

(Montessori, 2007), nos describe el material didáctico de la siguiente manera: No es un simple pasatiempo, ni una sencilla fuente de información, es más que eso, es material didáctico para enseñar. Están ideados a fin de captar la curiosidad del niño, guiarlo por el deseo de aprender. Para conseguir esta meta han de presentarse agrupados, según su función, de acuerdo con las necesidades innatas de cada alumno.

El sentido de esta investigación se resume a una sola frase: más recursos significa más oportunidades de desarrollo.

Los niños necesitan de mediadores que les ayuden a integrar el conocimiento y los juegos para niños de preescolar conjuntan la alegría del juego con la didáctica: teoría y práctica de un tema. Por eso, los materiales educativos para la Educación Inicial son el mejor recurso para consolidar sus aprendizajes porque materializan el conocimiento, ayudándoles a ejercitar las habilidades que ya tenían y a adquirir nuevas.

Según (Cortés, Navarrete y Troncoso, 2009), nos indican sobre los estímulos en los niños: Cada vez que los niños(as) usan alguno de sus sentidos (vista, tacto, olfato, audición, gusto), se crea una nueva conexión. Esto quiere decir que, si al niño(a) se le provee de diversas experiencias en forma continua, se estará favoreciendo las conexiones cerebrales que se generan a partir de los estímulos de dichas experiencias, transformándose así, en la base de los futuros aprendizajes que pueda adquirir el infante, convirtiéndose en una herramienta clave para dicho proceso y una consideración que no debe escapar de los educadores infantiles. 
Los estudios realizados por los autores, los niños/as deben desarrollarse en un ambiente agradable y lleno de estímulos, caso contrario los aprendizajes serán limitados. Si la maestra parvularia no es quien le da la posibilidad al niño de sumergirse a un nuevo mundo, nadie más se la va a dar. El análisis de esta investigación me lleva a confirmar que muchas instituciones carecen de buen material didáctico y que además no todas las maestras tienen los estudios necesarios para llevar a cabo una verdadera enseñanza.

Según (Barca, Almeida,Porto-Rioboo,Peralbo \& Brenlla, 2012), en la investigación realizada en su artículo científico, nos indican afirmando que todos los resultados son: Las principales variables motivacionales y de estrategias que afectan al rendimiento académico, de manera positiva o negativa, proporcionan información fehaciente para poder determinar cómo el profesorado puede apoyarse, en su función docente, en aquellas variables de estrategias de aprendizaje de organización, comprensión, en las estrategias de apoyo, así como en las de autoeficacia ya que, sin duda, facilitarán la motivación para el aprendizaje y, en definitiva, para el rendimiento académico.

La motivación escolar es inherente a los procesos enseñanza - aprendizaje, migrando a nuevos procedimientos basados en experiencias previas las cuales permitan planear nuevos procesos que favorezcan el descubrimiento, la construcción y apropiación de nuevos conocimientos por parte de los educandos, empleando para ello estrategias flexibles y modificables de acuerdo con las características de lo que se aprende, y muy especialmente, de quién aprende.

Realmente es la maestra quien debe ser la motivadora y promotora de nuevos aprendizajes y la institución quien aporte con la ayuda de todo el material didáctico posible. En este mismo sentido, actualmente se habla de un aprendizaje más dinámico con los infantes, puesto que su mayor atracción es el juego, por lo tanto, la motivación y la planificación de las clases deben girar en torno a ello, es decir, buscar que, a través del juego e interacción con los materiales didácticos, el estudiante adquiera las habilidades requeridas en su proceso formativo.

(Rodríguez, 2012), en su ensayo abordo que: Son ambientes de aprendizaje, que elementos lo constituyen, sobre las condiciones necesarias para su creación y el papel que juega el facilitador al propiciar ambientes de aprendizajes idóneos al desarrollo de las competencias de los estudiantes.

La función del profesional es crear un clima de seguridad y confianza, manteniendo relaciones personales positivas con alumnado y familias, con el objetivo de conseguir que sea eficaz el "ambiente de aprendizaje" que les ofrece. ¿Cómo? Seleccionando y organizando materiales, distribuyéndolos en el espacio, organizando el tiempo, con propósitos educativos permitiendo la mayor implicación del alumno/a en su propio aprendizaje, gestionando democráticamente.

Por otro lado, los estudios también permitieron afirmar que los materiales didácticos no se limitan al enriquecimiento o evaluación de los saberes transmitidos, sino que son un soporte de ese proceso de aprendizaje didáctico o dinámico. Por ello, es por lo que debe ser pensado el 
currículo como una oportunidad para el estudiante. Es evidente, entonces, que el material didáctico en el aprendizaje del ser humano es sumamente importante, más, cuando se trata de procesos de formación en la primera infancia, debido a que en esta etapa los niños requieren ambientes gratos y estimulantes, que propician nuevos saberes y posibiliten un mejor desarrollo en todas sus dimensiones.

Según (Ministerio de Educación, 2014) sugiere lo siguiente: Es necesario ofrecer a los niños muchas oportunidades para ejercitar cada destreza. Se requiere abundante práctica. Con una vez nunca será suficiente. Por ello, las destrezas deben trabajarse de manera consistente durante varios días, semanas $\mathrm{y}$, en ocasiones, durante meses hasta que los niños consigan dominarlas, practicando en diversos ambientes y situaciones (pág. 34)

Un niño no puede aprender las destrezas de nadar, cantar o dibujar a través de escuchar a su maestra. Las destrezas se aprenden "haciendo", "practicando", "ejercitando".

No hay más verdad que ésta. La experimentación los lleva a palpar la realidad de otra manera. Se usan muchas veces todos los sentidos para realizar una actividad y eso es imprescindible para su desarrollo integral. Se ha aprendido que el ambiente y las dinámicas, que se generan en el aula, permiten que los estudiantes tengan la suficiente autoconfianza como para dar su opinión respecto a los varios temas o varias situaciones que puedan surgir en el aula de clase.

\section{Metodología}

Según (Palella \& Martins , 2010), nos define: La Investigación de campo consiste en la recolección de datos directamente de la realidad donde ocurren los hechos, sin manipular o controlar las variables. Estudia los fenómenos sociales en su ambiente natural. El investigador no manipula variables debido a que esto hace perder el ambiente de naturalidad en el cual se manifiesta.

La investigación fue de campo y se tomó como muestra a 36 niños. Fue llevada a la práctica con el fin de describir y explicar las dificultades que se presentan en el trabajo académico a nivel de aula del SubInicial 1 y 2 de Educación Inicial de la escuela "Diana Belén", Periodo 2017 ubicada en el norte de la ciudad de Guayaquil, sobre todo resaltar la importancia del material didáctico en el aprendizaje significativo de los estudiantes.

Para la demostración del trabajo se llevaron a cabo procesos de observación y práctica acerca del uso efectivo del material didáctico y, además, si este existía, cual era. Dentro de la observación, se utilizó un diario para las varias anotaciones.

Nivel de la investigación:

$\checkmark$ Descripción del alumnado:

Estudio del comportamiento de cada estudiante durante la motivación en las dinámicas.

$\checkmark$ Descriptiva, explicativa:

Proponer la aplicación de nuevos recursos didácticos para el aprendizaje en niños y niñas del SubInicial 1 - 2 de Educación Inicial.

Métodos de investigación:

- Método deductivo: 
Los métodos deductivos son los que tradicionalmente más se utilizan en la enseñanza, lo cual queremos cambiar incrementando los métodos experimentales y participativos

- Método inductivo:

Es el ideal para lograr principios, y a partir de ellos utilizar el método deductivo.

Normalmente en las aulas se utiliza primero el método deductivo.

- Método analógico o comparativo:

Es fundamental la forma de razonar de los más pequeños, sin olvidar su importancia en todas las edades.

El material didáctico que se propuso fue preparado previamente; de consecuencia, las clases fueron siempre activas y complementadas por una actividad que no estaba específicamente mirada o reducida a un simple lápiz y un papel.

Se buscó también salir del aula de clase y experimentar, ya que dentro no había muchos recursos, ni mucho menos rincones para cada actividad. Se trató de compensar con varias actividades para mantener ocupados a los niños y niñas. Se realizó un video fue una de las actividades que se propuso para que los niños experimenten con sus pies las diferentes texturas de los materiales. (Esteves, 2018)

\section{Resultados}

El resultado que arrojaron las encuestas hechas a los niños fue positivo. La puesta en escena de varias actividades motivó a los niños a querer practicar siempre algo nuevo. Fueron estimulados de manera correcta con diferentes materiales. Todo era novedoso para ellos y sentían curiosidad por descubrir su uso y, sobre todo, si ellos podían usar el material. Poco a poco fueron ganando confianza y se apropiaron de cada nueva experiencia y su respectivo aprendizaje.

Importante es resaltar la predisposición que los estudiantes tenían al momento de ejecutar las órdenes. Cumplían con todo y se encontraban muy animados y felices. Cabe recalcar que el niño ve cada actividad como si fuera un juego y es justamente eso lo que el docente debe aprovechar. El niño no sabe que detrás de ese "juego" existe o fue programado para un aprendizaje específico y enriquecedor. El niño es curioso y hay que aprovechar sus características y rasgos propios de su edad para animarlo a que querer aprender más. La maestra a su vez se vio muy gratificada por los resultados que sus niños alcanzaron.

En cada clase, la diversidad de materiales que se usaron fue siempre diferente y la reacción de los niños fue muy positiva. Lo tomaban como nuevos "juegos" con nuevos objetos. La prueba final para plasmar el aprendizaje la superaron con éxito.

\section{Discusión}

En la hipótesis se planteó si tenía mucha incidencia el uso del material didáctico y efectivamente pude corroborar y ahora afirmar que más recursos se tienen a disposición y mejor será el resultado de lo que se quiere enseñar. No necesariamente hay que recurrir a una hoja, pegamento y papel para enseñar varias cosas. Existen otras maneras y materiales que se pueden utilizar que hacen de la labor docente también algo no siempre monótono y aburrido, se podría osar decir. 
La escuela en cuestión contaba muy poco material y la maestra se basaba en lo "típico". En su labor se notaba su predisposición, pero no la acompañaba de mucha creatividad ni mucho menos tomaba ideas nuevas. Tampoco usaba muchas técnicas ni estrategias de enseñanza, lo cual era un poco negativo para el aprendizaje. La institución no permitía a las maestras experimentar mucho y su pedagogía de enseñanza se basaba mucho en el modelo tradicional-conductista. Sin embargo, con la nueva propuesta que se llevó, se notó un cambio rotundo en la maestra y los estudiantes. Ambos estaban activos y listo para enseñar la maestra y los niños aprender de manera casual y experimentaría.

\section{Conclusión}

Los recursos materiales, entorno, contexto físico, equipamiento, materiales didácticos y curriculares, procedimientos y estrategias son el soporte instrumental que hace posible el proceso de enseñanza-aprendizaje.

Niñas y niños aprenden y se desarrollan poniendo en marcha los siguientes procedimientos, entre otros: manipulación, observación multisensorial, descubrimiento, exploración, experimentación, interacción con iguales y adultos cercanos, juego. Es difícil el desarrollo y el aprendizaje sin: motivación intrínseca, propósito, compromiso, libertad, espontaneidad, entrega, esfuerzo. La acción facilita los procesos de asimilación propios del aprendizaje. El juego y la acción libre superan la contradicción esfuerzo-placer que tanto dificulta la entrega del alumno en la escuela. La interacción con los iguales, la mediación del educador y el juego son situaciones que permiten anticipar y ensayar comportamientos más avanzados.

John Locke manifiesta que el niño es como una pizarra en blanco donde no hay nada escrito, sus caracteres pueden ser moldeados. De hecho, nos encontramos de frente a una realidad muy diferente cuando de niños se trata. Comencé escribiendo en esta investigación que nos encontrábamos con una nueva sociedad y que todo lo que se haga tiene que ser para encaminarla y aprovecharla al máximo. Aunque el sistema escolar no sea el mejor y trate de expandirse igualmente y no tenga siempre el apoyo "económico" de la institución donde se ejerce, debemos recordad que los niños son como esponjas absorbentes y que está en nuestras manos guiar a los futuros hombres del mañana.

Es un arduo trabajo que muchas veces no es reconocido ni mucho menos remunerado por su gran importancia y larga incidencia en la sociedad; sin embargo, hemos escogido la labor por vocación y de esto depende mucho como ejecutemos nuestro trabajo que debe ser siempre impecable y "mejor del anterior".

\section{Bibliografía}

Alvarado, A. (6 de Enero de 2017). Xperiencias De Aprendizaje Según Elcurriclo Inicial. Obtenido de SlideShare: https://es.slideshare.net/AnabelAlvarado2505/experiencias-de-aprendizaje-segnel-currculo-inicial

Barca, Almeida,Porto-Rioboo,Peralbo \& Brenlla. (s.f de s.f de 2012). Motivación escolar y rendimiento: impacto de metas académicas, de estrategias de aprendizaje y autoeficacia. Obtenido de http://www.redalyc.org: http://www.redalyc.org/html/167/16723774023/ 
Cortés, Navarrete y Troncoso. (s.f de s.f de 2009). Construyendo Experiencias Desde La Temprana Infancia. Obtenido de Sistema nervioso: una mirada general: http://repositorio.uchile.cl/tesis/uchile/2009/cs-troncoso_m/pdfAmont/cs-troncoso_m.pdf

Esteves, Z. (2018) Trabajo de Campo. Video. https://www.youtube.com/watch?v=23DMhEhmor8

Guerrero \& Idrovo. (s.f de s.f de 2010). Estudio Del Material Didactico De La Metodologia De Rincones Lúdicos En Educación Inicial. Obtenido de El material didáctico en la educación inicial: http://dspace.ucuenca.edu.ec/bitstream/123456789/2330/1/tps630.pdf

Guerrero Duran M. E., Idrovo Argudo S. M. (2010). Estudio del material didáctico de la metodología de rincones lúdicos en educación inicial (Tesis). http://www.educomunicacion.es/figuraspedagogia/0_montessori.htm

M. Montessori, El Método Montessori, (1912). Currículo Educación Inicial, 2014. Ministerio de Educación.

Manrique Orozco, A. \& Gallego Henao, A, M. (2012), El material didáctico para la construcción de aprendizajes significativos (Tesis).

Ministerio de Educación. (s.f de s.f de 2014). 3.4.5 Sugerencias para lograr un óptimo desarrollo de las destrezas. En E. T. Básica, Guía Metodológica Para La Implementación Del Curriculo De Educación Inicial (págs. 1-58). Quito: s.e. Obtenido de 3.4.5 Sugerencias para lograr un óptimo desarrollo de las destrezas: https://educacion.gob.ec/wpcontent/uploads/downloads/2015/05/Guia-Implentacion-del-curriculo.pdf

Montessori, M. (s.f de s.f de 2007). La pedagogía de la responsabilidad y la autoformación. Obtenido de La importancia de María Montessori: http://educomunicacion.es/figuraspedagogia/0_montessori.htm

Palella \& Martins . (s.f de s.f de 2010). Tipos de Investigación. Obtenido de Investigación de Campo: http://planificaciondeproyectosemirarismendi.blogspot.com/2013/04/tipos-y-diseno-de-lainvestigacion_21.html

Rodríguez, H. (s.f de s.f de 2012). Universidad Autónoma del Estado de Hidago. Obtenido de Ambientes De Aprendizaje: https://www.uaeh.edu.mx/scige/boletin/huejutla/n4/e1.html

Varios Autores, 2017/08/31, Debates y escenarios en el campo educativo. CIDE ECUADOR (centro de investigación y desarrollo Ecuador)

Varios Autores, Memorias científicas del I congreso internacional en la Educación Inicial y Básica. CIDE Ecuador (centro de investigación y desarrollo Ecuador) https://docs.wixstatic.com/ugd/54b18d_a0c8054eba5a4386940ed08b85f7ac22.pdf 